\title{
Equidad de género en la gran minería del cobre de Chile. Experiencias de inserción laboral femenina en espacios masculinizados ${ }^{1}$
}

\author{
Pablo Salvador Gómez Fuentealba ${ }^{2}$ \\ Nicolás Angelcos Gutiérrez ${ }^{3}$
}

Fecha de recepción: 06 de marzo de 2018

Fecha de aprobación: 14 de mayo de 2018

\begin{abstract}
Resumen
Este artículo analiza los alcances y obstáculos de las políticas de inserción femenina en la gran minería del cobre en Chile. La incorporación de las mujeres se realiza en un sector estratégico de la economía, tradicionalmente dominado por varones, con remuneraciones y prestigio sobre la media nacional. Nuestra hipótesis es que, pese a la eliminación de fronteras institucionales que limitaban la participación de las mujeres, subsisten fronteras simbólicas (Lamont y Bail, 2005) que dificultan el alcance de la equidad de género. Así, analizamos dos fronteras que operan como obstáculos a la incorporación efectiva de las mujeres: estereotipos laborales asociados a lo masculino y femenino, y asociación entre mujer y madre.
\end{abstract}

Palabras clave: Chile, maternidad, minería, equidad de género, fronteras simbólicas.

\section{Gender equity in the large copper mining industry in Chile. Experiences of female labor insertion in masculinized spaces}

\begin{abstract}
This article analyzes the scope and obstacles of female insertion policies in the large copper mining industry in Chile. The incorporation of women
\end{abstract}

Los autores agradecen al Centro de Estudios de Conflicto y Cohesión Social, CONICYT/FONDAP/15130009. Además, se agradece el apoyo por los contactos en la División El Teniente, a partir del estudio previo "Inserción laboral de mujeres en sectores estratégicos de la economía chilena: un fenómeno emergente", cuya investigadora responsable fue Patricia Retamal, y los contactos de la División Chuquicamata proporcionados por la tesista en sociología Camila Muci.

2 Magíster en Sociología, Universidad Alberto Hurtado. Santiago, Chile. Contacto: pablosalvadorg@gmail.com.

3 Doctor en Sociología de la École des Hautes Études en Sciences Sociales de París. Universidad Andrés Bello. Universidad de Chile. Santiago, Chile. Contacto: nicolas. angelcos@unab.cl. 
takes place in a strategic sector of economy, traditionally dominated by men, with payments and prestige over the national average. Our hypothesis is that, despite the elimination of institutional boundaries limiting women's participation, symbolic boundaries remain (Lamont and Bail, 2005) hindering the gender equity. We analyze two barriers that operate as obstacles to the effective incorporation of women: labor stereotypes associated to masculine and feminine, and association between woman and mother.

Keywords: Chile, motherhood, mining, gender equity, symbolic borders.

\title{
Equidade de gênero na grande mineração do cobre do Chile. Experiências de inserção laboral feminina em espaços masculinizados
}

\begin{abstract}
Resumo
Este artigo analisa os alcances e obstáculos das políticas de inserção feminina na grande mineração do cobre no Chile. A incorporação das mulheres realiza-se em um setor estratégico da economia, tradicionalmente dominado por homens, com remunerações e prestígio sobre a média nacional. A nossa hipótese é que, apesar da eliminação de fronteiras institucionais que limitavam a participação das mulheres, subsistem fronteiras simbólicas (Lamont e Bail, 2005) que dificultam o alcance da equidade de gênero. Assim, analisamos duas fronteiras que operam como obstáculos na incorporação efetiva das mulheres: estereótipos laborais associados ao masculino e feminino, e associação entre mulher e mãe.
\end{abstract}

Palavras-chave: Chile, maternidade, mineração, equidade de gênero, fronteiras simbólicas.

\section{Introducción}

Durante las últimas décadas, a partir de diversos tratados y acuerdos internacionales, los países de América Latina han llevado a cabo una serie de políticas de Estado en pos de la incorporación de las mujeres al mercado de trabajo formal (Abramo y Valenzuela, 2006). Los gobiernos latinoamericanos han identificado a las mujeres como sujetas prioritarias de la política pública, asumiendo la necesidad de incentivar acciones para potenciar su escolarización y posterior profesionalización, con el objetivo de impulsar procesos de liderazgo y empoderamiento femenino en los diversos espacios político-sociales.

El empoderamiento femenino ha sido concebido como uno de los elementos centrales para la superación de la pobreza, 
el mejoramiento de la calidad de vida y los procesos de desarrollo económico (OIT, 2002; Tomei, 2006). Tanto los gobiernos como las empresas transnacionales han asumido un discurso de "equidad de género", posibilitando la incorporación de la población femenina a actividades productivas y, en casos específicos, a labores tradicionalmente asociadas a los varones. Con ello, las políticas de género han asumido la labor de visibilizar y dar cuenta de los "beneficios" que obtienen las empresas al incorporar masa femenina calificada, considerando la transformación de las lógicas al interior de los equipos de trabajo, contribuir al desarrollo personal-familiar de las mujeres y crear espacios en condición de igualdad y valorando la diversidad (Albornoz, 2017).

Las empresas conciben la necesidad de integrar fuerza laboral femenina, argumentando que la acción trae consigo una serie de avances que posibilitan mantener el carácter competitivo de las empresas en un contexto global (Díaz, 2014). Dicha práctica fortalece el reconocimiento de un abanico de características asociadas a la construcción de la feminidad, como la afectividad, empatía, coordinación, entre otras, vinculadas preferentemente a los imaginarios de género movilizados al interior del espacio minero (Salinas et al., 2010).

El presente artículo tiene como intención describir y analizar el proceso de inserción femenina en la actividad minera en dos casos específicos: las divisiones El Teniente y Chuquicamata, ambas administradas por la empresa Codelco, propiedad del Estado de Chile. La importancia de los casos se refiere a que se trata de un rubro estratégico en la economía chilena, históricamente desempeñado por varones, y que presenta niveles de remuneración y prestigio por sobre la media nacional. Por ello, contrasta con los estudios que destacan la incorporación de la mujer a trabajos precarizados y mal remunerados.

La hipótesis de nuestro artículo es que, pese a la eliminación de las fronteras institucionales que limitaban la incorporación de 
la mujer al trabajo minero hasta un periodo muy reciente ${ }^{4}$, hoy se mantienen y fortalecen fronteras simbólicas (Lamont y Bail, 2005) que permiten la reproducción del trabajo minero a través de jerarquías culturales masculinas, al interior de las cuales las mujeres tienen una posición de subordinación. Dentro de éstas, la producción y reproducción de estereotipos asociados al trabajo masculino y femenino, además de la identificación de la mujer como madre, constituyen los mayores obstáculos para la integración y reconocimiento pleno de las mujeres mineras como trabajadoras. Frente a este escenario, la conciliación entre trabajo y familia -tensión constante en la incorporación femenina al mercado laboral- aparece como un problema de carácter privado que, aunque cuente con ciertos soportes institucionales, es responsabilidad de las mujeres su solución. Esta tensión será central para entender la experiencia de las mujeres en su incorporación al trabajo minero.

En primer lugar, se enmarcará teórica y metodológicamente el artículo; en segundo, se caracterizará el contexto relativo a las políticas de inserción de la mujer al mercado laboral y las políticas específicas desarrolladas por Codelco, y, finalmente, se identificarán las fronteras simbólicas que limitan la integración y reconocimiento de las mujeres en el trabajo minero.

\section{Identidad, orden de género y fronteras simbólicas}

En diversos estudios contemporáneos (Honneth, 1997; Ricoeur, 2004; Fraser, 2005) se ha posicionado la categoría de "reconocimiento social" como un eje central para comprender la

\footnotetext{
4 Hasta 1996, el Código del Trabajo prohibía la participación de la mujer en las faenas mineras, relegándolas a actividades de administración o domésticas. El artículo 15 del Código del Trabajo establecía: “Las mujeres no podrán ser ocupadas en trabajos mineros subterráneos ni en faenas calificadas como superiores a sus fuerzas o peligrosas para las condiciones físicas o morales propias de su sexo".
} 
formación de identidades individuales y colectivas. En esta dirección, siguiendo los trabajos pioneros de B. Moore, E. P. Thompson o C. Taylor, se ha destacado la importancia de la dimensión moral (Fassin y Lézé, 2013) para entender los conflictos sociales, señalando la centralidad que tiene la percepción de ciertos principios de justicia compartidos para comprender la movilización colectiva. En otras palabras, los individuos tendrían mayor disposición a participar de una acción colectiva en la medida en que perciban que ciertos principios de justicia, que consideran legítimos, han sido violados (Honneth, 1997; Merklén, 2009; Kokoreff y Lapeyronnie, 2013).

Tal como destacan Michèle Lamont y Chrisopher A. Bail (2005), los estudios recientes sobre política del reconocimiento son expresivos de una crisis del liberalismo, en la medida en que ponen el acento en la dimensión intersubjetiva que está a la base de la construcción de la identidad individual. Para los grupos dominantes, el cuestionamiento de las jerarquías culturales por parte de los grupos dominados pone en cuestión las identidades que han construido históricamente a través de los siglos.

Para entender los rasgos internos y externos en la producción de identidades colectivas, siguiendo a Jenkins, Lamont plantea la necesidad de estudiar la interacción entre los procesos de identificación del grupo y las formas de categorización social: "por una parte, los individuos deben ser capaces de diferenciarse de los otros fundándose sobre criterios comunes y sobre un sentido de pertenencia compartida en el seno de su grupo. Por otra parte, para que una identidad colectiva objetiva emerja, ese proceso de identificación interno debe ser reconocido por las personas exteriores al grupo" (Lamont y Bail, 2005, p. 64).

Siguiendo este propósito -distinguir los límites internos y externos en la conformación de identidades colectivas-, Lamont ha propuesto el concepto de "fronteras simbólicas". A partir de una relación crítica con la obra de P. Bourdieu, quien identifica a priori los límites entre grupos desde sus conceptos de "capital" y "cam- 
po" (Lamont, 2008), Lamont destaca la necesidad de comprender el sentido que los individuos dan a sus diferencias y si éstos las perciben como modificables o no. Así, la autora define las fronteras simbólicas como "las categorías mentales comunes a diversas poblaciones, concerniendo lo que define las similitudes y las diferencias internas y externas a los grupos, y más específicamente cómo los individuos utilizan sus herramientas cognitivas para determinar qué grupos son iguales y por qué, cómo categorías de individuos pueden ser medidas a partir de estándares definidos que permitan la comparación" (Lamont y Bail, 2005, p. 66).

Estas fronteras no se limitan a aspectos socioeconómicos; también comprenden aspectos culturales y morales. En oposición a la sociología de la dominación de P. Bourdieu, las fronteras no solamente contribuyen a reproducir el orden, sino que también pueden ser movilizadas para combatir las fronteras sociales entre grupos (Lamont, 2008). En este sentido, el avance en el reconocimiento pleno de las mujeres como trabajadoras supondría el quiebre con las fronteras simbólicas que las subordinan.

Ahora bien, tal como señala la autora, este análisis no solo es productivo para el estudio de la identidad colectiva en su relación con los movimientos sociales, tal como destacamos inicialmente, sino también para el análisis de la experiencia cotidiana de los individuos. En este sentido, a partir del análisis de las fronteras simbólicas entre trabajadores y trabajadoras al interior de la minería, podremos identificar las jerarquías culturales que sostienen la cultura masculina de la minería y que estarían siendo cuestionadas por la inserción de la mujer a este rubro. De este modo, la experiencia de inserción de mujeres al mundo de la minería no puede ser comprendida sin entender los principios de clasificación con que históricamente se ha justificado su exclusión.

A este respecto, es importante recordar que el mundo minero constituye un espacio altamente masculinizado, no solo por la mayoritaria participación de varones al interior del rubro, sino también por las prácticas e interacciones cotidianas y laborales 
construidas en torno a imaginarios que configuran lo "masculino" y lo "femenino". La faena minera, como espacio productivo, cuyos saberes y conocimientos se encuentran vinculados a la ciencia y tecnología, se posiciona como un espacio incompatible para lo concebido como "femenino", dado que exige cualidades propias de la identidad "masculina", tales como: conocimiento matemático, capacidad de abstracción, interés por la competencia, entre otros (Arango, 2006). La segregación de espacios y conocimientos por sexo se concibe como una operación necesaria para la mantención de la división sexual del trabajo (Benería, 1981), perpetuando la diferenciación de labores y funciones sociales para varones y mujeres, fortaleciendo lo "masculino" con lo productivo y lo "femenino" con lo reproductivo. El sistema sexo/género (Rubin, 2013) define las actividades y labores de varones y mujeres al interior del espacio minero, a partir de destacar y profundizar las diferencias biológicas y fisiológicas de los cuerpos.

El cuerpo de los "hombres" pareciese ser el apropiado para las labores asociadas a la minería, lo que deviene en categorizar como propiamente "masculino" las labores vinculadas a la fuerza física o la capacidad cognitiva en relación con conocimientos matemáticos e ingenieriles. Es así como la diferencia sexual materializada en los cuerpos constituye "una forma primaria de relaciones significantes de poder" (Scott, 2013, p. 290), permitiendo la construcción de símbolos culturales para representar los modelos de lo femenino/masculino e instalando interpretaciones normativas que delimitan prácticas, discursos y acciones de cada sexo. El orden de género se posiciona como un modelo de organización de expectativas, prácticas, referencias y acciones de cada sexo distribuido de manera jerárquica, constitutivo de la identidad subjetiva de varones y mujeres.

\section{Metodología}

Este artículo forma parte de la investigación “Disposiciones y resistencias subjetivas a la participación sindical en mujeres de la 
Gran minería del cobre en Chile", financiada por la Universidad Diego Portales a través de su Fondo Semilla (Folio n063-2014), cuyo investigador responsable es el coautor de este artículo.

En este proyecto se realizaron veinte entrevistas en profundidad a trabajadoras mujeres de las divisiones El Teniente y Chuquicamata de la empresa minera estatal Codelco, con distintas posiciones al interior de la estructura de poder de la empresa (operarias, supervisoras, profesionales). Paralelamente, se entrevistó a un dirigente sindical hombre, cuyo sindicato contiene el mayor número de trabajadoras mujeres de la División El Teniente.

Las entrevistas de la División El Teniente fueron realizadas por los autores del artículo en la ciudad de Rancagua. Las personas entrevistadas fueron contactadas a partir de otras trabajadoras que participaron en un estudio previo 5 . Las entrevistas en la División Chuquicamata las realizó la tesista de pregrado en sociología Camila Muci, directamente en la faena. Estos contactos fueron realizados a través de una gerente de la empresa. Todas las entrevistas fueron realizadas en 2015.

Las entrevistas se analizaron de forma inductiva, creando categorías a partir de ellas. Para garantizar la integridad de las personas entrevistadas, se utilizó un consentimiento informado aprobado por el comité de ética de la Universidad Diego Portales.

\section{Contexto en pos de la incorporación femenina}

La Cuarta Conferencia Mundial sobre la Mujer (1995) constituye un hito relevante para instalar la perspectiva de género al interior de las políticas de Estado. Actualmente, la Agenda 2030 para el Desarrollo Sostenible (Ministerio de Desarrollo Social,

\footnotetext{
5 "Inserción laboral de mujeres en sectores estratégicos de la economía chilena: un fenómeno emergente". Investigación financiada por la Facultad de Ciencias Sociales de la Universidad de Chile, cuya investigadora responsable fue Patricia Retamal.
} 
2017) incorpora la igualdad de género como un objetivo en sí mismo, siendo la oportunidad para la superación de las desigualdades de género que se reproducen al interior de espacios económicos, civiles y políticos. Los desafíos que plantea la Agenda 2030 dialogan con las demandas históricas en pos de la igualdad de género, motivando que tanto los Estados como las empresas incidan e incentiven acciones en el marco de garantizar iguales derechos a las mujeres y a los varones. Las medidas e iniciativas buscan la modificación de marcos jurídicos, la eliminación de las múltiples formas de violencia hacia las mujeres, el reconocimiento y valoración del trabajo de cuidados y labores domésticas no remuneradas, y la participación efectiva de las mujeres en espacios de decisión política y estratégica.

Una de las propuestas más importantes, propiciada por la Cuarta Conferencia Mundial sobre la Mujer y considerada por las iniciativas contemporáneas en torno a la igualdad de género, es la necesidad por incidir en la elaboración de políticas públicas y sociales, considerando espacios de disputa en pos de la incorporación transversal del tema de género en las agendas de los gobiernos (Guzmán, 2001), proceso denominado "gender mainstreaming" (Rodríguez, Mora y Navarro, 2011). La inclusión de la perspectiva de género de manera transversal en las acciones del Estado ha propiciado la transformación de la arquitectura institucional, incorporando estrategias y metodologías acordes a los objetivos en torno a la igualdad de género. La aplicación del concepto conlleva la visibilización de diversos conflictos al interior del espacio de trabajo, instalando desafíos al momento de pensar una política con perspectiva de género (Álvarez, 2010).

A partir de las transformaciones motivadas por la paulatina inclusión de la perspectiva de género en el quehacer institucional, se incentiva la aplicación de programas y medidas estandarizadas, concibiendo la necesidad de incorporar a las mujeres a espacios político-sociales. Es así que la implementación de programas de inclusión para el mejoramiento de la distribución de 
la riqueza y el aumento de la participación social del crecimiento económico, se encuentra ligada a la incorporación de diferentes sujetos al mercado laboral, posibilitando la integración comercial de la región al mercado mundial. Durante los últimos años se “aprecia voluntad política para la incorporación de medidas tendientes a la eliminación de la discriminación y el logro de la equidad de género en el trabajo en cada país, se refleja el tratamiento de los aspectos sociales y laborales en los acuerdos de integración comercial y regional" (Oxman, 2006, p. 235).

En síntesis, a partir de la intención de eliminar la segmentación horizontal y vertical por género al interior del mercado laboral (Anker, 1997), los convenios internacionales buscan la eliminación de los elementos de discriminación mediante acciones afirmativas, estimulando agentes económicos, como lo son las mujeres, y posibilitando la generación de una mayor rentabilidad familiar y así el mejoramiento de la calidad de vida, junto con la formulación de estrategias en pos de la constitución de un nuevo capital humano.

\section{Políticas de inserción femenina en Codelco}

La gran minería del cobre en Chile constituye el espacio productivo fundamental para el desarrollo económico del país ${ }^{6}$, dado que, a partir de la extracción del material cuprífero, se sustentan las políticas sociales desarrolladas por el Estado. Por otro lado, el trabajo en minería constituye una labor de prestigio y estabilidad salarial, lo que permite la construcción de trayectorias sociales estables y en ascenso social para trabajadores/as.

Basada en fronteras institucionales restrictivas para las mujeres, la minería se había mantenido, a lo largo de las décadas,

\footnotetext{
La participación de la minería en el PIB chileno se ubica en torno al 9\%, con amplias variaciones entre algunos años de la década de los noventa $(7 \%)$ y de la segunda mitad de la década del 2000 (20\%) (Consejo Minero, recuperado de http:/ / www.consejominero.cl/chile-pais-minero/).
} 
como una actividad económica fundamentalmente masculina. Sin embargo, en el marco de las políticas generales descritas en la parte anterior, Codelco asumió la labor de contratación, atracción y selección de nuevo personal, registrando una alta dotación femenina para 2014, dando cuenta de un aumento significativo después de 15 años.

Si bien la participación laboral femenina ha aumentado en Chile, alcanzando el 48,6\% del total de la fuerza de trabajo disponible, sigue siendo un porcentaje bajo dentro del contexto latinoamericano $(52,8 \%$ ) y de los países de la OCDE $(63,8 \%$ ) (Abarca y Riquelme, 2015). Asimismo, su incorporación a labores históricamente masculinizadas, como es la minería, es también baja en comparación, por ejemplo, con Canadá y Australia, países con importante actividad minera, cuya dotación de mujeres alcanza en promedio el 16\% (Albornoz, 2017; Díaz, 2014). En Chile, la participación femenina sobre la dotación total en minería es del 8,6\% y menos del 1\% en relación a ocupar cargos de decisión (Albornoz, 2017). Frente a este escenario, el Estado chileno, a través de la empresa Codelco, decidió emprender una serie de iniciativas y acciones en pos de la incorporación de dotación femenina, obteniendo para noviembre de 2017 una participación del 9,3\% (Ídem.).

Las estrategias e iniciativas implementadas por Codelco, en su búsqueda por la igualdad de género al interior del espacio minero, se destacan por suscripción a programas y orientaciones generales (Programa Iguala-2007), modificación de normativa institucional y certificación del trabajo en torno a la igualdad de género y conciliación trabajo-familia (Norma Chilena 3262-2013), implementación de estrategia focalizada en diversidad de género, que contempla la generación de principios, directrices, indicadores y fomento al liderazgo femenino (2015), implementación del convenio de desempeño único y actividades de sensibilización (2016), y, finalmente, durante 2017, la generación de política corporativa de diversidad de género y conciliación, junto con un conjunto de precertificaciones en diferentes faenas. 
Los argumentos que orientan las políticas de inserción son heterogéneos: por un lado, para el SERNAM7 ${ }^{7}$, dicho aumento es de vital importancia, ya que, al ser una actividad económica con ingresos relativamente altos, permite avanzar en la equidad de género y disminuir así la brecha salarial entre varones y mujeres. Por otro lado, CODELCO concibe la incorporación femenina como un factor de aumento de la competitividad de la empresa, en el sentido de que potencia ciertas habilidades femeninas, tales como "mayor orientación hacia el autocuidado y la seguridad laboral, preocupación por mantener un buen clima organizacional, capacidad de dar calidez a los ambientes laborales, valorización de los equipos mixtos de trabajo, manejo de múltiples variables en forma simultánea, compromiso y lealtad con el trabajo, responsabilidad, dedicación y disciplina, habilidades en el manejo y cuidado de instrumentos y equipos de alta precisión, bajo nivel de ausentismo laboral, preocupación por la presentación personal" (Díaz, 2014, p. 63).

Finalmente, un tercer argumento, de corte más técnico, se vincula con la proyección en términos de déficit de mano de obra calificada que tiene el rubro. Según la Sociedad Nacional de Minería (Rodríguez, 2012), para 2015 se estimaba un déficit relativo a 23 mil trabajadores, lo que aumentaría a 69 mil si es que se considera la necesidad de trabajadores subcontratados. Esto se suma a la revolución tecnológica de los medios de producción en esta actividad económica, que ha permitido progresivamente superar a la fuerza física como recurso principal (Díaz, 2014).

Respecto de los resultados de la incorporación femenina en CODELCO, específicamente para los casos de la presente investigación, la División Chuquicamata es el centro con mayor dotación (505 trabajadoras) con un 8,13\%, seguido de el Teniente (235 trabajadoras) con un 4,78\% (Codelco, 2014). A pesar de ser los espacios productivos con mayor población femenina, son otras

\footnotetext{
7 "Programa de Buenas Prácticas con Equidad de Género".
} 
divisiones (al menos tres) las que cuentan con salas de extracción y almacenamiento de leche materna para las trabajadoras.

Como vemos, en un marco internacional favorable a la incorporación de la mujer al mercado laboral, la empresa estatal CODELCO, especializada en la extracción de cobre, ha decidido implementar numerosas iniciativas orientadas a incentivar la contratación de mujeres en un rubro tradicionalmente ocupado por varones. Lo fundamental de este proceso es que la actividad minera es un empleo formal con alto estatus y remuneraciones por sobre la media nacional, lo que contrasta con la incorporación mayoritaria de mujeres a empleos flexibles, precarios y mal remunerados (Montecinos y Arenas, 1999; Staab, 2012).

A continuación, profundizaremos en las fronteras simbólicas que limitan este proceso y que son parcialmente enfrentadas por las políticas desarrolladas por la empresa.

\section{Trabajo y jornada minera: obstáculo en la experiencia femenina}

El trabajo minero se ha asociado históricamente al mundo masculino, dado que, como labor productiva, solo podía ser ejercido por varones. A partir de ello, la incorporación femenina en el inicio de la actividad minera se encontraba ajena a la faena, insertándose en ocupaciones que se concebían como una extensión de las labores domésticas al interior del campamento minero. La inserción femenina en el campamento constituía así un dispositivo de regulación de la sexualidad (Klubock, 1995), situando a las mujeres en tareas tales como el servicio doméstico, lavandería o cuidado.

Por otro lado, el carácter masculino del trabajo al interior de la faena minera se ha asociado históricamente a elementos como fuerza física, destreza y resistencia, la posibilidad de desplazamiento a espacios inhóspitos para la vida y extensas jornadas laborales, asociadas a un segmento subalterno de trabajadores; por 
otro lado, al pensamiento lógico-racional, al intelecto y el saber técnico-científico, ligados a un segmento dirigente de trabajadores. Lo anterior constituye un sistema de símbolos y representaciones que sustentó la exclusión explícita de las mujeres de la faena minera (Troncoso, 2014).

Hoy los resguardos legales que impedían el ingreso de las mujeres a las faenas mineras no existen, pudiendo considerarse que se han eliminado las barreras explícitas; sin embargo, aún persisten elementos, de carácter implícito, constitutivos de la denominada "cultura minera" (Pavez y Hernández, 2014), que se levantan como obstáculos no solo para la inserción, sino que también para las experiencias y trayectorias de las mujeres ya dentro del trabajo en minería. Algunos de estos elementos han sido abordados en la literatura, entregando pistas de las dinámicas y relaciones sociolaborales en las que se insertan las mujeres en minería, otorgando énfasis en diferentes dimensiones, tales como: la identificación de modelos femeninos al interior del trabajo minero (Stefoni, 2005); estrategias de protección y valoración por parte de las mujeres frente la cultura minera (Troncoso, 2014); asignación de jornadas, posiciones jerárquicas y remuneraciones asociadas al trabajo femenino (Díaz, 2014), y las caracterizaciones y prejuicios que se asumen del trabajo femenino en espacios masculinizados (Ayala, 2012).

A partir de las entrevistas realizadas y de la experiencia abordada por la literatura, a continuación se exponen dos fronteras simbólicas del trabajo minero que constituyen posibilitadores y obstáculos de la experiencia femenina en un rubro masculinizado, elementos que no son ampliamente consignados al momento de elaborar los programas y políticas de incorporación de la mujer en este sector productivo. En primer lugar, se presentan los mecanismos y prácticas que permiten la segregación material y simbólica al interior del trabajo minero, y, en segundo lugar, las estrategias y experiencias de las mujeres en la búsqueda por conciliar los proyectos laboral y familiar. 


\section{¿Y ella sabe lo que es el trabajo en minería? Expectativas, características y función de las mujeres}

Como ya señalamos, la segregación de la mujer al interior del trabajo minero se expresó no solamente mediante prejuicios y estereotipos asociados al trabajo femenino, sino que se institucionalizó como una barrera legal a su acceso, consignada en el Código del Trabajo hasta 1996. Derogada esa barrera legal, quisiéramos interrogar, en este apartado, las fronteras simbólicas que permiten mantener las jerarquías culturales masculinas, pese a la incorporación de las mujeres a este rubro.

Dentro de estas fronteras, la creación de estereotipos asociados al trabajo femenino y masculino es central. Lo interesante de su utilización es que permite, en primer lugar, justificar la participación de la mujer en la minería, espacio que históricamente no le correspondería. De este modo, si bien el uso de estereotipos permite reforzar las fronteras entre hombres y mujeres, también permite abrir un espacio simbólico para su incorporación, el cual no existía hasta hace algunas décadas. La incorporación de las mujeres al rubro minero se encuentra, así, fomentada en el reconocimiento de habilidades, capacidades y oportunidades que permitirían dinamizar y constituir nuevas relaciones al interior de la organización laboral. Así lo narra una dirigenta:

Hay estudios que demuestran que dándole más participación y más espacio a las mujeres dentro de estos grupos de trabajo, eeh la mujer también aporta, no aporta solamente en que sepa hacer la pega, sino que en el equipo, no sé po' las mujeres son más ordenadas, son más preocupadas, que no existan riesgos de accidentes".

(Dirigenta intersindical, División El Teniente)

Como vemos, esta dirigenta, en vez de cuestionar estos estereotipos laborales, los moviliza a su favor para justificar "científi- 
camente" (hay estudios...) el aporte específico que supone la participación de las mujeres en esta actividad. En la misma dirección, otra trabajadora señala:

Mira, al principio entré como secretaria técnica, en oficina técnica, y ahí más que nada se gestionaba (...) y ahí yo me acoplé mucho a esa área porque organizaba la oficina, (...) tenía harta pega por la auditorías, y todo el tema, y ahí estaba yo plantada, mostrando todo, mostrando todo mi trabajo, todo lo que se hacía, cómo se hacían los procesos, cómo se gestionaba, y como mujer que uno es más ordenada, normalmente uno apoya más ese lado, el lado del orden. (Proyectos y control de la operación global, División El Teniente)

Tanto el orden como el liderazgo afectivo, la disciplina y la responsabilidad se asumen como características femeninas que otorgan legitimidad a la inserción de las mujeres en el espacio masculino, dado que históricamente las relaciones laborales entre varones se conciben como conflictivas y competitivas. Tal como plantea Salinas (2007), los discursos masculinos en este tipo de áreas productivas movilizan concepciones de género, a la vez que se encuentran tensionados por nuevas concepciones y prácticas. En este caso, una lógica empresarial que, a través de valores heteronormativos, fomenta la inserción de la mujer en la minería por ser portadora de virtudes favorables a su desempeño laboral.

Ahora bien, si la identificación de ciertas habilidades femeninas asociadas a la gestión y administración del trabajo minero constituyen un elemento que permite justificar su presencia en la faena minera, el terreno mismo aún constituye un bastión masculino, en el cual las habilidades asociadas a la femineidad, tales como la delicadeza, la empatía y la debilidad, son utilizadas como argumento para reforzar la discriminación de género.

... viejos que te gritaban, yo andaba en terreno con otra compañera y las mujeres no tienen que estar aquí. Y también los mismos trabajadores menos reticentes con el 
tema, no entendían que una mujer les fuera a decir cómo hacer las cosas. (Gestión ambiental, División El Teniente)

Como opuesto a la delicadeza, la empatía y la debilidad que caracterizarían el comportamiento femenino, la fuerza física aparece como un rasgo marcadamente masculino y que sería definitorio del trabajo minero, especialmente de aquél desarrollado en la faena. Las grandes maquinarias de carga y traslado, la utilización de herramientas y el manejo de explosivos se encuentran vinculados simbólicamente al quehacer masculino en este espacio ${ }^{8}$. El trabajo en terreno implica el desgaste, agotamiento y exposición física, en condiciones de falta de higiene y comodidad que son aparentemente incompatibles con el trabajo femenino.

En las operaciones, en algunos casos, no todos, no está la tecnología en todos lados, todavía existen trabajos muy rudimentarios que requieren de mucha fuerza física para desarrollarlo y las mujeres no tienen desarrollado al nivel del hombre la fuerza física, entonces les cuesta mucho más, entonces eso hace que la socia, la niña o la trabajadora, se sienta que no puede, se sienta que no es capaz, los mismos compañeros la exigen mucho, y comienzan a ser problemas, sistemas que duran, o trabajos que duran un minuto, treinta segundos con un trabajador, la trabajadora lo realiza en un minuto y medio, eso hace que se empiece catalogar que no sirve. (Dirigente Sindical, División El Teniente)

Para complementar, las trayectorias profesionales y disciplinares al interior de la ciencia y la tecnología han sido construidas históricamente y de manera constante como espacios incompatibles para la población femenina, debido a considerarse incompetentes frente a labores, técnicas o en el ejercicio de fuerza física. Wajcman (1991) analiza la exclusión de las mujeres a partir de la construcción sociohistórica de la ciencia y la tecnología como parte fundamental en la reproducción de la división sexual del trabajo. Los rígidos patrones de la diferenciación sexual en el mundo del trabajo sentaron las bases de la marginación de las mujeres en la utilización y control de la tecnología. El mundo masculino ha negado o regularizado la participación de las mujeres en los ámbitos de creación, uso, utilización e innovación de tecnología, asentándose así como uno de los pilares y enclaves "masculinos"(Arango, 2006). 
La pretensión de maximizar las utilidades y la disminución de los tiempos de producción interpela de manera negativa a las mujeres que comienzan a insertarse al trabajo minero, ya que su labor se encuentra cruzada por menores condiciones para el trabajo en terreno; les es asignada la condición de "aprendices", teniendo que haber un tiempo de acoplamiento de la población femenina con el rubro, mientras que se considera que los varones se encuentran asociados naturalmente al trabajo. Finalmente, aunque los avances tecnológicos permiten que las mujeres puedan responsabilizarse en áreas del trabajo productivo, estos al parecer no se encuentran masificados, dado que no constituyen una necesidad para la población masculina.

Los espacios segregados al interior de la organización laboral en minería -resultado de la diferenciación histórica entre labores y actividades asociadas a varones y mujeres- se encuentran reforzados por la representación simbólica de lo femenino, cuyas características, tales como ser afectivas, cálidas, indefensas, permiten el ejercicio de la labor de manera distinta a los varones. Esta diferencia es traducida en términos de relaciones familiares, lo que es valorado por las trabajadoras, ya que la dominación que estructura este espacio se expresa en actitudes de cuidado por parte de los trabajadores hombres.

Es que igual tener una mujer entremedio, no todos los varones reaccionan de la misma forma, pero todos son como más acogedores, como si tú fueras su hija, ¿cachai? Sobre todo si trabajai con viejos mayores // la relación con el jefe igual es diferente. Porque igual el jefe te trata como de, como no sé, como más como su hija// yo creo como que te ven como, como que te ven como su hija, como que te tratan de apoyar, de guiar. (Mantenedora eléctrica, División El Teniente)

En su posición de debilidad, a partir de un contexto desfavorable para el transitar femenino, las mujeres trabajadoras son apadrinadas por sus pares masculinos, sobre todo por los varo- 
nes con mayor trayectoria y jerarquía. Las relaciones labores de carácter asimétrico entre varones y mujeres, tanto a nivel vertical como horizontal, son representadas a partir de la homologación de las dinámicas familiares. Por ello, las mujeres se asumen como "hijas", desvalidas frente al rubro y solas, a partir de la convivencia mayoritariamente con varones.

Yo, como era chica, siempre fui como la hermana menor. Nosotros somos como una familia, tení que verlo así, estamos nueve horas allá arriba, de lunes a viernes, en las cuales comenzai a conocer a las personas, etc. Te encariñai con mucha gente, cuando yo llegué era la única mujer que estaba en la oficina, tanto en una oficina técnica como en topografía, trabajaba en ambos lados. Entonces vi pasar mucha gente, muchos varones dentro de esas oficinas, la rotación de personal, etc. (...) el hecho que fuera tan chiquitita, fui criada por ellos, entonces a la larga me benefició, de alguna manera, porque me hice más fuerte, me hice más fuerte en ese ámbito, yo nunca tuve problemas con varones, con los chicos nada por lo menos, ni un drama, tampoco de que 'Ay, que me gusta él', de amoríos, no, nunca, fui siempre bien seriota pa' mis cuestiones. (Proyectos y control de la operación global, División El Teniente)

Situadas a partir de la denominación de "hermanas" o "hijas", las mujeres posibilitan su inserción en el rubro, dado que las relaciones familiares, conducentes a roles aparentemente asexuados, constituyen el soporte para asegurar respeto y validación por parte de sus pares masculinos. Los lazos afectivos y emocionales benefician a las mujeres, porque permiten establecer espacios de seguridad en los que la desconfianza, los "amoríos", la envidia o la competencia no se establecen como criterios para constituir relaciones entre pares.

En síntesis, podemos ver cómo la creación y movilización de estereotipos asociados al trabajo femenino y masculino consti- 
tuyen fronteras simbólicas que, en ciertos momentos, permiten la integración y legitimación de la mujer en la actividad minera, como en el caso de la gestión y la administración, pero que, en otros, refuerzan su segregación, como en el caso del trabajo en terreno. Estas fronteras no son solamente producidas y reproducidas por varones, sino que también por las mujeres, quienes las utilizan para justificar su posición dentro del orden minero. El traslado de lógicas familiares al interior del trabajo -basadas en una relación paternalista entre trabajadores varones con trayectoria y mujeres jóvenes recién ingresadas- facilita la relación entre distintos géneros, al mismo tiempo que reproduce la posición de subordinación que tienen las mujeres.

En el siguiente apartado profundizaremos en una frontera simbólica transversal a la participación de la mujer en el mercado del trabajo, a saber, la distinción y conciliación entre el trabajo remunerado y el trabajo reproductivo.

\section{Y si tú trabajas, ¿quién cuida a los niños? Conciliación trabajo-familia}

Parte importante de la literatura especializada otorga una valoración positiva a la inserción de las mujeres al mercado del trabajo minero, ya que constituye una posibilidad para mejorar sus trayectorias de vida, otorgando independencia económica y autonomía individual. Sin embargo, el modelo familiar y las dinámicas al interior del hogar obligan a la población femenina a generar estrategias de negociación y conciliación entre las responsabilidades que demanda el trabajo y los deberes impuestos por el cuidado de los/as hijos/as.

Considerando la reflexión de Silvia Berger (2010), mientras cabe destacar las iniciativas y acciones que abogan por la equidad de género e igualdad de condiciones en el mercado laboral frente al ordenamiento androcéntrico, por otro lado es posible problematizarlas y cuestionarlas en torno a su escasa incidencia en la desarticulación de la familia patriarcal, que continúa limi- 
tando la participación de las mujeres como fuerza de trabajo libre, debido a la obligatoriedad de las labores de cuidado.

Por lo mismo, las mujeres, en cualquiera sea el sector o posición en que se encuentren trabajando, se hallarán constantemente asociadas al ejercicio y práctica del trabajo reproductivo, lo que se denomina como "doble jornada laboral" (Friedan, 1983), intensificado a partir del modelo de organización productiva en minería, dado que este se encuentra estructurado sobre la base de largas y demandantes jornadas de trabajo, dificultando el acuerdo entre vida laboral y responsabilidades familiares. A este respecto, un dirigente sindical nos "recuerda" cuál es el rol que le corresponde "naturalmente" a la mujer:

La mujer, recuerda, que es la base de nuestra sociedad, tu mamá, mi mamá, la mamá de todos los varones, es como la influencia que tiene el hijo o la hija es su mamá. Entonces una mamá ausente no sé cuál es el desarrollo que va tener el niño en su infancia, ¿cachai? Con una mamá ausente, con una mamá minera, que le está dando el colegio, las zapatillas, la bicicleta, pero, pero ¿quién está criando al niño ahí?, con una mamá ausente. Eeeh, yo creo que no es, no es, no veo muy bien, yo creo que debiéramos tener normas que resguarden a las mujeres de esta minería, o sea, entra a minería pero sin hacer turnos de noche, entonces que tú estés en tu casa con tus niños, que duerma contigo, para crear ese lazo, esa afectividad que debiera tener su hijo con su madre (...) Entonces, en el ámbito de la sociedad, yo creo que no estoy muy de acuerdo a que la mujer entre a trabajar a la minería, así tan desprotegida, debiera haber más protección de los turnos, debiera haber más protección en las áreas que pueda entrar y que no pueda entrar, eeeh, entonces por ahí estoy un poco en desacuerdo, aunque busco la igualdad de género. (Dirigente sindical, División El Teniente) 
El espacio minero se configura como espacio masculino, dado que la distribución y organización de los tiempos de trabajo impiden el ejercicio de la crianza y el cuidado al interior de la esfera familiar; dicha ausencia en el hogar aparentemente se puede conceder a los jefes de hogar, proveedores económicos del núcleo doméstico; sin embargo, resulta imposible disociarlo de la labor de las mujeres. Por ende, la iniciativa de incorporar a las mujeres a la minería, desde la óptica masculina, no puede ser a costa de la crianza y acompañamiento de la formación de los niños/as, apelando a las aptitudes socioafectivas que "naturalmente" poseen las mujeres. Finalmente, la inserción laboral femenina tiene como expectativa la reestructuración de las relaciones sociales al interior de la minería; sin embargo, también constituye una pretensión la inmutabilidad de los roles dispuestos en la familia, por lo que la maternidad representa una frontera simbólica que limita las trayectorias de inserción femenina y desarrollo en el rubro minero.

[Las mujeres] han estado ausentes con sus hijos, tienen pocos permisos. Le cuesta entender al supervisor que la mamá se pueda ausentar porque no llegó la persona que le cuidaba los niños en la noche, y que llamí al supervisor que no llegó la persona y que te vai a tener que quedar; eeeh el supervisor, al final -pone que tiene mucho problema la señora, la niña para trabajar-y va poniendo ausencia, ausencia no mah, no le da ni vacaciones. (Dirigente sindical, División El Teniente)

Las experiencias de las mujeres se encuentran constantemente significadas como exitosas o fracasadas a partir de la posibilidad de ajustar o acoplar sus trayectorias laborales con la organización de los proyectos familiares, donde la norma de género impone y establece sobre las mujeres la responsabilidad del cuidado, crianza y enseñanza en la esfera doméstica. Dichas negociaciones individuales entre la esfera pública del trabajo y la esfera privada del hogar parecieran ser producto de la desigual organización social del tiempo, permeada por relaciones de género asimétricas, que requieren de la participación de estructuras institucionales 
que posibiliten amortiguar las demandas y deberes domésticos, permitiendo adecuar los tiempos productivos y reproductivos (Yopo, 2015, p.105).

La inserción de las mujeres se encuentra mediada por el ejercicio de la maternidad, pudiendo constituir un obstáculo para su incorporación formal en minería.

La disposición que tengan las empresas a generar el abrir las puertas a más mujeres, porque, por ejemplo, en las faenas, eeh, para una empresa pudiera decir: ... si coloco mujeres, si son más de veinte, tengo todo el tema de las salas cuna, eeh, si están con el tema del posnatal tengo que darle permiso para el tema de lactancia. (Dirigenta intersindical, División El Teniente)

La presencia de salas cuna al interior de las empresas es un elemento importante que permite la conciliación entre el trabajo productivo y el reproductivo desarrollado por las mujeres. Es por ello que la disposición de las mujeres a trabajar en minería se asocia directamente a su presencia:

Yo me había venido con una guagua de seis meses, en el camino me enteré que había una sala cuna, la guagua se fue a la sala cuna y, en fin, se fue dando todo muy fácil, por eso digo que para nosotros no fue complicado, se nos dieron las cosas, y estar en una sala cuna era, pero... la sala cuna cumplía todas mis expectativas. (Supervisora, División Chuquicamata)

La inserción de la población femenina en espacios masculinizados ha obligado a otorgar facilitadores institucionales que permitan generar trayectorias sin mayores conflictos para la población femenina, teniendo una actitud "consciente" de las necesidades de las mujeres respecto de la labor por cumplir en sus hogares. Salir de la casa, para las mujeres, o el abandono del hogar, para los varones, constituye una decisión difícil de tomar para las "madres" o "esposas", puesto que pone en riesgo la estabilidad de los proyectos familiares, como también se desligan del proce- 
so de enseñanza en la primera infancia de sus hijos/as, cediendo la facultad orientadora y afectiva a otras mujeres (empleadas, familiares o educadoras de párvulos).

Las mamás debieran estar hasta los tres años, tres-cuatro años con sus hijos, cachai? Por lo menos bajar temprano, no te estoy diciendo que faltí al trabajo, sino que bajar temprano, cachai, que igual la ley dice que hasta los dos años, o al año de amamantamiento y todo el tema, tú tienes el derecho de bajar temprano y todo el tema, nosotras trabajamos mucho allá arriba, muchas horas, a eso me refiero. Entonces, lo mejor tener esa, ser racional y decir: "sabi qué, esta mina (mujer) es buena, sabe hacer su pega (trabajo) y la necesitamos, puta démosle la oportunidad, que la gestión la haga, no sé po, desde las nueve que entra hasta las cuatro", porque hay muchos tiempo muertos también entre todos los procesos, y yo creo que nosotras trabajaríamos también con mucha más motivación, ¿cachaí? Yo creo que sería, no sé po', valoraríamos más lo que estamos haciendo, hoy en día la mujer lo valora, profesionalmente, a uno que le gusta la pega, $y$ todo el tema, ¿cachaí? Lo hacemos, pero también vemos que hay ciertas injusticias pucha digo yo, no sé po', tengo una compañera que tuvo dos hijos seguidos, ¿cachaí? ¿Cómo sería para esa madre? a lo mejor por lo menos hasta los cuatro años que el cabro chico entra al jardín y toda la cuestión, bueno podría ser, servir, son unas horas más que estai con tu hijo, que lo veí, que trabajai como más motivada. (Proyectos y control de la operación global, División El Teniente)

A partir de la ausencia de espacios institucionales o contractuales, como es el caso de la División El Teniente, las demandas de género o las exigencias de las mujeres trabajadoras se encuentran permeadas por la maternidad, evidenciando la contradicción que interpela a las mujeres, al participar de un rubro de prestigio y valoración social que le exige disposición completa, mientras 
tiene que desempeñar de manera funcional los mandatos sociales al interior de la familia. Es tal la tensión entre ambos espacios que, en ciertos casos, se generan argumentos críticos respecto de la supuesta utilización "estratégica" que harían las mujeres de la maternidad.

Yo creo que hacen falta más mujeres, pero mujeres que estén dispuestas a trabajar, porque muchas llegan y se embarazan, y la imagen de la mujer acá queda como por los suelos (...) entonces yo soy crítica en ese aspecto. Si tú llegas a trabajar tienes que mostrarte y tratar de hacer todo lo posible para no embarazarte, para que te contraten. (Jefa de turno, División Chuquicamata)

En razón de esta tensión, las iniciativas institucionales, como las presentes en la División de Chuquicamata, que permiten el ejercicio de la maternidad, son altamente valoradas por las mujeres trabajadoras, dado que posibilitan la crianza de los hijos/as en espacios asociados a la minería, acomodándose a los tiempos y ritmos del trabajo minero.

En ese tiempo, nosotros en Chuqui, teníamos una sala cuna en Chuqui, entonces me pasaban a buscar y yo partía a trabajar con mis hijos. Al mayor lo tuve un año en la sala cuna y al segundo lo tuve casi dos años. Pero teníamos la sala cuna, entonces nos pasaban a buscar, los dejábamos en la sala cuna, nos íbamos a trabajar y después los pasábamos a buscar; si había que amamantar salíamos a amamantar y si no, en la tarde, los pasaba a buscar para devolverte a Calama. (Analista en gestión, División Chuquicamata)

De todas formas, a pesar de la existencia de espacios institucionales, la maternidad se despliega como frontera simbólica en las diferentes dimensiones del ejercicio minero, dado que las tareas o labores domésticas implican la resolución de problemas ajenos a la faena y, con ello, inciden en la productividad de las trabajadoras. El embarazo, la lactancia, el cuidado, las licencias, 
la enseñanza y hasta la menstruación son elementos que dificultan el ejercicio laboral de las mujeres, generando trayectorias intermitentes dentro del rubro.

Yo nunca quise quedar embarazada, pero no por un tema de que no quisiera (...) una de las desventajas que sí considero y que encuentro son las mujeres que trabajan y tienen hijos, por ejemplo, tengo muchas amigas que tienen hijos de dos años, cuatro años y, no sé po', su hijo se enferma y se vuelven locas, porque el hecho de que, claro que no están abajo, está la nana (trabajadora de casa particular), nadie cuida, uno, o sea, nadie va cuidar al hijo como lo cuidai tú (...), a veces la gente no se pone en el lugar de uno como mamá -los varones-, uno como mamá la preocupación que tiene de dejar a su hijo todos los días en la casa, yo creo que esa es una de las cosas también por lo cual nunca quise tener un hijo, estando trabajando, por ese simple hecho, de que me condicionaba mucho estar arriba, muchas horas. (Proyectos y control de la operación global, División El Teniente)

La maternidad es concebida como una responsabilidad obligatoria de las mujeres, lo cual exige una jornada laboral altamente flexible para el "sacrificio" del cuidado, lo que la jornada y el trabajo minero no posibilitan, provocando que las mujeres posterguen la decisión de ser madres, por ser visto como problemático. Sin embargo, los mandatos sociales al interior de la familia no sólo interpelan a las mujeres en relación a su función de madres, sino también en relación con la compatibilidad con la pareja (esposo o conviviente).

Él hacía turnos diez por cinco, igual yo no lo veía en diez días po', cuando estaba de noche, porque él se iba día y noche, cuando estaba de día lo veía un rato en las tardes, y los fines de semana cuando él estaba de noche, nos veíamos un rato en la mañana, y la nada porque él tenía que dormir, así que fue complicado y, en realidad, 
como estaba trabajando, era estudiante, aparte estaba casada, fue como súper pa' mí como mujer, fue súper complicado, porque él era jefe de turno en ese tiempo, y la verdad, es que ya se había titulado y todo el tema, era yo la que se llevaba todo el peso, de ver el hogar, y además la familia externa, que es la familia tuya, que es la familia de la que tú vení, que es tu mamá y hermanos, que también me hice mucho tiempo cargo de mi hermano más chico, que estaba estudiando, entonces no, no. (Proyectos y control de la operación global, División El Teniente)

La decisión de las mujeres acerca de la maternidad constituye una tensión respecto de la proyección profesional, tanto en el ascenso laboral en la jerarquía minera como en la jornada laboral. La población femenina que se inserta en minería tiene como pretensión la validación de sus conocimientos y prácticas, otorgando importancia a la legitimidad de su trabajo. El alto nivel de exigencia, (auto) impuesto a partir de las condiciones del rubro minero, exige a las mujeres construir trayectorias sólidas e ininterrumpidas, por lo que la maternidad y la crianza se presentan como un obstáculo.

Yo me embaracé de mi tercer niño, bueno (...) me embarazaron, porque yo no quería (risas), y bueno ahí problemas (...) bueno, de hecho, yo no quería tomar el posnatal porque me aburría en la casa, me obligaron porque me dijeron que legalmente tienes que hacerlo. (Jefa de turno, División Chuquicamata)

Los intereses personales, la movilidad profesional y el proyecto familiar tensionan la experiencia femenina en minería, porque en las mujeres recae la responsabilidad de las labores reproductivas. Por esta razón, la modificación de las jornadas, la flexibilización de los tiempos de trabajo así como el apoyo familiar en el cuidado de los niños/as o la acción institucional con la protección del posnatal y sala cuna, constituyen mecanismos y 
estrategias para la conciliación de la vida familiar y laboral, permitiendo trayectorias significadas positivamente por parte de las mujeres trabajadoras.

Puedes trabajar y puedes tener bebé, puedes hacer todo, tú no dejas de ser mujer femenina si estás en un mundo de la minería, no... tienes que seguir siendo femenina $\mathrm{y}$ tienes que seguir haciendo lo que hace una mujer. Lo tienes que complementar no más po', pero ese complemento te lo da tu pareja, te lo dan tus hijos, te lo da tu familia, en general, que se adapten, o buscar los mejores espacios para poder lograrlo. (Gerente de extracción, División Chuquicamata)

Ahora bien, a pesar de que las mujeres trabajadoras reconozcan y signifiquen sus experiencias como positivas en el proceso de inserción al trabajo minero, reconociendo apoyos institucionales, como las salas cunas, en el caso de la División Chuquicamata, o mecanismos legales para la protección de la maternidad, como el pre y posnatal, las problemáticas familiares se instalan como obstáculos para la realización profesional de las mujeres, dado que sus labores en la mina se encuentran entrecruzadas con las responsabilidades de crianza y maternidad.

El desarrollo personal (...) profesional se contrapone contra eso, pero como ya decidí, como ya tomé un camino y lo que uno tiene que hacer es adaptarse, o sea, todo va de la mano, todo va en ordenarse, y yo quiero hacer las dos cosas bien, pero eso (...), las dos cosas y bien. No la una y dejar (...) la una de lado por la otra, no. (Mecánicos PALAS, División Chuquicamata)

A partir del imaginario de género y de la imposición masculina que otorga la exclusiva responsabilidad de las labores reproductivas a las mujeres, tanto las empresas de minería como la organización laboral al interior de la mina se desligan de dichos problemas y consideran que las mujeres los resuelven. Cuando las mujeres se integran al mercado laboral los varones no se in- 
corporan paralelamente en el mundo del cuidado (Tobío, 2012). Así, la tarea por conciliar el espacio laboral y familiar produce tensiones generadas a partir de su inserción laboral, las cuales son privatizadas e individualizadas en las mismas mujeres.

Las trabajadoras asumen como propios los obstáculos y la resolución de los mismos, reconociéndose como punto de conflicto al tensionar el proyecto familiar o la maternidad a partir de la decisión por profesionalizarse.

Y te dicen "no, no puedo estudiar porque tengo un bebé", y yo lo he escuchado, entonces tú dices "pero, ¿cómo? si tener un bebé no es un impedimento para poder estudiar", depende de ti no más, de nadie más. Ahora, si tú te pones ese impedimento de que "no puedo estudiar porque estoy embarazada o porque tengo un bebé", las barreras te las pones tú, no te las ponen las demás personas, no te las pone la sociedad. (Gerente de extracción, División Chuquicamata)

La adaptación, organización de los tiempos y los riesgos que se atribuyen a la trayectoria profesional al interior de la minería dependerían de las estrategias y decisiones de las mismas mujeres, en pos de favorecer sus procesos de inserción en la organización minera. Tal como fue decidir ingresar a un rubro masculino, la dificultad de la conciliación entre trabajo y familia constituye un obstáculo relevante, pudiendo presentarse tanto una experiencia exitosa como fallida.

Ya cuando salí a trabajar fue una etapa difícil, pero de ahí en adelante en realidad había que hacerlo. Pero sí, fue difícil ahí el tema de las nanas, el tema de los traslados, eh... el tema de no haber podido de alguna manera compartir muchas cosas que, como mujer, madre uno se lo merece (...) Pero, cuando ella se tituló, porque ella estudió enfermería, ella una cosa que agradece es eso. O sea, nunca ha sentido que yo le falté, porque de algu- 
na manera uno supo estar. (Dirigenta sindical, División Chuquicamata)

En la experiencia de las trabajadoras, la naturalización y asimilación de las dificultades que trae su incorporación al rubro minero, como el trabajo en un ambiente masculino, los constantes mecanismos de validación y legitimidad de las labores realizadas, las dificultades para conciliar tiempos de trabajo y crianza, el desapego afectivo del espacio familiar, las estrategias para consolidar la maternidad, entre otros, se presentan como un costo y parte del riesgo asumido por las mujeres, dado que sus réditos son la mejora de la calidad de vida a partir de ingresos, y el reconocimiento de un ejercicio laboral al interior de una actividad con prestigio social.

En síntesis, podemos ver cómo la conciliación entre trabajo y familia es experimentada por las mujeres como una conciliación entre trabajo productivo y reproductivo, en la que la responsabilidad de los varones está prácticamente ausente. Es decir, como plantea Yopo (2016), el tiempo de las mujeres se organizaría de acuerdo con el rol subordinado que desempeñan tanto en la esfera doméstica, familiar, como en la esfera laboral. La distinción entre mujer trabajadora y mujer madre opera como una frontera simbólica que tensiona la trayectoria de inserción femenina en este rubro, en la que embarazarse aparece, a momentos, como una mala decisión al interior de una actividad con alto estatus y remuneraciones. Frente a esta tensión, la generación de condiciones materiales para la conciliación (salas cuna, permisos de lactancia, cambio en el sistema de turnos) es altamente valorada por las mujeres, aun cuando asuman finalmente que es una responsabilidad individual y no familiar o social la articulación entre trabajo y familia.

\section{Reflexiones finales}

En este artículo hemos querido problematizar la incorporación de la mujer a la actividad minera, a partir del estudio de dos divisiones de la empresa estatal CODELCO. Nuestro objetivo 
ha sido poner en tensión, no tanto el horizonte normativo de la equidad de género, sino cómo su implementación se enfrenta a la existencia de fronteras simbólicas que limitan el éxito de políticas públicas orientadas a la incorporación de la mujer al trabajo, en condiciones de alto prestigio y remuneraciones por sobre la media nacional.

Hemos demostrado cómo, a pesar de la eliminación de las fronteras institucionales que limitaban la incorporación de la mujer al trabajo minero, aún subsisten fronteras simbólicas que impiden su integración efectiva. Mostramos, a su vez, cómo estas fronteras no solo implican limitaciones a la integración de las mujeres, sino que también pueden ser movilizadas por éstas para abrir un espacio simbólico que, hasta hace algunas décadas, les era negado. Por una parte, la generación de estereotipos asociados al trabajo masculino y femenino permiten justificar su incorporación en las labores de gestión y administración, pero limitan su participación en el trabajo de terreno, caracterizado aún por la fuerza física. Esto último es importante, ya que los varones justifican su posición de dominación por su exposición física en el trabajo desempeñado. Por otra parte, el ejercicio del trabajo reproductivo por parte casi exclusiva de la mujer es una tensión permanente que impide el reconocimiento pleno de las mujeres mineras como trabajadoras.

Las trayectorias y experiencias femeninas se encuentran tensionadas por la maternidad, en la cual las mujeres asumen como propias las dificultades en la labor de conciliar sus proyectos profesionales con los familiares, por lo que exponen e indican de manera ambigua su satisfacción en la resolución de dichos obstáculos. A partir de ello, reconocen de manera importante la participación de la institucionalidad minera, en su acción por proveer servicios de cuidado, como la sala cuna, o el respeto al ejercicio de la maternidad, al igual que el apoyo familiar en el reemplazo de la mujer en las tareas o labores domésticas. Sin embargo, la individualización y privatización de aquellas problemáticas en las mujeres trabajadoras termina por invisibilizar la importancia 
del trabajo reproductivo en el funcionamiento de la vida social (Ávila, 2013). De esta manera, no se reconoce la responsabilidad y deber de la empresa, ni tampoco la de sus pares masculinos en las labores reproductivas al interior del hogar.

Las políticas o acciones en pos de la inserción laboral femenina en el espacio minero, a pesar de que permiten desarticular la segregación ocupacional, no problematizan las consecuencias que se atribuyen en la asignación exclusiva y absoluta de las mujeres trabajadoras a la labor de crianza y enseñanza al interior de la esfera familiar, restándole importancia a una de las dimensiones que presenta, de manera explícita o implícita, uno de los principales obstáculos en las trayectorias profesionales.

Lo anterior, de ninguna manera constituye una invitación a detener o impedir la incorporación femenina en minería, sino a visibilizar que las actuales relaciones laborales y las decisiones institucionales reproducen las nociones e imaginarios de género, asumiendo como espacios aislados las esferas laboral y familiar, cuando de manera contraria estas se encuentran interseccionadas. Las labores privadas o domésticas no solo se atribuyen al hogar y la familia, sino que se presentan de manera permanente en las experiencias femeninas, incorporándose al interior de la mina, superando los límites de "lo personal". Coherente por lo planteado por Yáñez (2004), surgen varias interrogantes sobre este tipo de iniciativas y acciones, dado que, en el marco de las presentes innovaciones y transformaciones de un espacio históricamente masculinizado, hasta qué punto las políticas implementadas se deben restringir al ámbito y fronteras asociadas al trabajo remunerado, cuando los hallazgos dan cuenta de que uno de los principales soportes de las desigualdades de género se encuentra en las otras formas de trabajo, siendo principalmente las actividades domésticas y de cuidado, sin además considerar las actividades político-sindicales, que siguen siendo otro bastión masculino (Angelcos, 2015).

Finalmente, a pesar de los avances declarados por las trabajadoras de la División Chuquicamata y de los retrasos expresados en 
la División El Teniente, las políticas institucionales de las empresas mineras aún se presentan como formas de solucionar problemáticas de "las mujeres" o de "las madres", excluyendo a la población masculina en la responsabilidad de la crianza y la familia, reforzando con ello el imaginario de género. La política de "equidad de género" propuesta por parte de la institucionalidad no asume el nudo problemático y de conflicto que presenta la distribución desigual de labores y funciones entre varones y mujeres, por lo que no es posible denominarla como política de género capaz de reorganizar las relaciones de poder, sino que se expresa como una acción de discriminación positiva hacia las mujeres, que reproduce y mantiene la obligatoriedad de la maternidad en las mismas.

\section{Referencias}

Abarca, M. y Riquelme, V. (2015). Más mujeres en los sindicatos. Sectores con alta sindicalización femenina. Santiago: Dirección del Trabajo, Gobierno de Chile. Recuperado el 15 de noviembre 2017, de http://www.dt.gob.cl/documentacion/1612/ articles-106799_recurso_1.pdf.

Abramo, L. y Valenzuela, M.E. (2006). Inserción laboral y brechas de equidad de género en América Latina. En L. Abramo (Ed.), Trabajo decente y equidad de género en América Latina, Santiago: Oficina Internacional del Trabajo (OIT). Recuperado el 15 de noviembre 2017, de http://www.institutouejn. nqnwebs.com/documentos/genero/Trabajo\%20decente $\% 20 \mathrm{y} \% 20$ equidad $\% 20 \mathrm{de} \% 20 \mathrm{~g} \% \mathrm{C} 3 \%$ A 9 nero $\% 20$ en\%20America \%20Latina.pdf

Albornoz, L. (2017) Diversidad de género en minería: ¿cómo damos el siguiente paso? En: Gender Summit 12 para América Latina y el Caribe-CONICYT, Recuperado el 30 de mayo de 2018, de http:/ /www.conicyt.cl/gendersummit12/wp-content/ uploads/2017/12/1-Laura-Albornoz-Conicyt.pdf

Álvarez, A. (2010). El mainstreaming de género y sus nuevos desafíos: Repensando el concepto de igualdad(es). Revista del CLAD Reforma y Democracia, 47, 1-13. Recuperado el 15 de noviembre 2017, de http://old.clad.org/portal/publicaciones-del-clad/revista-clad-reforma-democracia / articulos/047-junio-2010/alonso 
Angelcos, N. (2015) Disposiciones y resistencias a la participación sindical en mujeres de la Gran Minería del cobre en Chile. Documento del trabajo ICSO, 21. Santiago, Chile: Universidad Diego Portales.

Anker, R. (1997). La segregación profesional entre hombres y mujeres. Repaso de las teorías. Revista Internacional Del Trabajo, 116(3), 343-370.

Arango, L. (2006). Género e ingeniería: la identidad profesional en discusión. Revista Colombiana de Antropología, 42, 129-156. Recuperado de: http: / / www.icanh.gov.co/?idcategoria=1404

Ávila, M. B. (2013). Presentación Mesa de discusión sobre trabajo doméstico. Revista de Estudios Sociales, 45, 229-232. DOI: https://doi.org/10.7440/res45.2013.19

Ayala, E. (2012). Mujer y minería. Situación de la industria chilena. Tesis de magíster, Universidad de Chile. Recuperado el 15 de noviembre 2017, de http://desarrolloproveedores.cl/dp/ wp-content/uploads/2012/11/Mujer-y-Mineria-Elizabeth-Ayala-20121.pdf

Benería, L. (1981). Reproducción, producción y división sexual del trabajo. Mientras Tanto, Icaria Editorial, 6, 47-84.

Berger, S. (2010) América Latina, la crisis y el feminismo. Pensando junto con Nancy Fraser, En: A. Girón (Coord.) Crisis económica: una perspectiva feminista desde América Latina. Caracas: CLACSO/IIE/UCV.

CODELCO. (2014). Recursos humanos. Memoria Anual, 112-122. Recuperado el 15 de noviembre 2017, de https://www. codelco.com/memoria2014/site/artic/20150406/asocfile/20150406162158/memoria_2014_recursos_humanos.pdf

Díaz, E. (2014). Mujeres en trabajos de hombres: segregación ocupacional y condiciones laborales en los sectores minería y construcción. Santiago: Departamento de Estudios de la Dirección del Trabajo. Recuperado el 15 de noviembre 2017, de http:/ / www. dt.gob.cl/documentacion/1612/articles-103028_recurso_1.pdf

Fassin, D. y Lézé, S. (2013). La question morale. Une anthologie critique. París: PUF.

Fraser, N. (2005). Qu'est-ce la justice sociale?: Reconnaissance et redistribution. París: La découverte, 
Friedan, B. (1983). La segunda fase. Barcelona: Plaza \& Janes.

Guzmán, V. (2001) La institucionalidad de género en el Estado: nuevas perspectivas de análisis. Serie Mujer y Desarrollo. Santiago de Chile: CEPAL/Naciones Unidas.

Honneth, A. (1997). La lucha por el reconocimiento. Por una gramática moral de los conflictos sociales. Barcelona: Crítica.

Klubock, T. (1992). Sexualidad y proletarización en la mina El Teniente. Proposiciones, 21, 45-54.

Klubock, T. (1995). Hombres y mujeres en El Teniente. La construcción de género y clase en la minería chilena del cobre, 1904-1951. En L. Godoy et al., Disciplina y desacato. Construcción de identidad en Chile. Siglos XIX (pp. 223-254) Santiago: Coedición SUR/CEM.

Kokoreff, M. y Lapeyronnie, D. (2013). Refaire la cité. L'avenir des banlieues. París: Seuil.

Lamont, M. (2008). Money, morals, and manners: The culture of the French and American upper-middle class. Chicago: University of Chicago Press.

Lamont, M. y Bail, C. (2005). Sur les frontières de la reconnaissance. Les catégories internes et externes de l'identité collective. Revue européene des migrations internationals, 21(2), 61-90.

Merklen, D. (2009). Quartiers populaires. Quartiers politiques. París: La Dispute.

Ministerio de Desarrollo Social. (2017). Informe de diagnóstico e implementación de la agenda 2030 y los objetivos de desarrollo sostenible en Chile. Santiago de Chile: Ministerio de Desarrollo Social, Consejo Nacional para la implementación de la Agenda 2030 para el desarrollo sostenible (ODS). Recuperado de: http:/ / www.chileagenda2030.gob.cl/storage/docs / Diagnostico-Inicial_2.0_Agenda2030-ODS_2017.pdf

Montecinos, V. y Arenas, A. (1999). The privatization of social security and women's welfare: gender effects of the Chilean reform. Latin American Research Review, 34(3), 64-73. Recuperado el 15 de noviembre 2017 de: http://www.jstor.org/stable/2503961

OIT. (2002). El ajuste laboral en América Latina: una perspectiva de género (1995-2002). OIT.

Oxman, V. (2006). Aspectos laborales y género en los acuerdos de inte- 
gración comercial y regional en las Américas. En L. Abramo (Ed.), Trabajo decente y equidad de género en América Latina, (pp. 233-262). Santiago: Oficina Internacional del Trabajo (OIT). Recuperado el 15 de noviembre 2017, de http:// www.institutouejn.nqnwebs.com/documentos/genero/Trabajo $\% 20$ decente $\% 20 \mathrm{y} \% 20$ equidad $\% 20 \mathrm{de} \% 20$ g\%C3\%A9nero\%20en\%20America\%20Latina.pdf

Pavez, J. y Hernández, G. (2014). Regímenes de trabajo, relaciones laborales y masculinidades en la gran minería del cobre (norte de Chile). En X. Valdés et al., Trabajos y familias en el neoliberalismo. Hombres y mujeres en faenas de la uva, el salmón y el cobre (pp. 169-263). Santiago: LOM.

Ricoeur, P. (2004). Parcours de la reconnaissance. Paris: Stocks.

Rodríguez, A. (2012). Mujeres en faena: La avanzada. Revista Qué Pasa Minería. Recuperado el 25 de noviembre 2016 de http:// www.quepasamineria.cl/index.php/vida-e-innovacion/item/254-mujeres-en-faena-la-avanzada

Rodríguez, M., Mora, C. y Navarro, C. (2011). El rendimiento de los mecanismos de participación: género y diseño institucional. Consejos de Distrito y Presupuestos Participativos en ciudades españolas. Revista del CLAD Reforma Y Democracia, 50, 254-259. Recuperado el 15 de noviembre 2017 de http:/ / www.redalyc.org:9081/articulo.oa?id=357533682005

Rubin, G. (2013). El tráfico de mujeres: notas sobre la "economía política" del sexo. En M. Lamas (Ed.), El género. La construcción cultural de la diferencia sexual (pp. 35-96). México: Programa Universitario de Estudios de Género: Instituto de Investigaciones sobre la Universidad y la Educación / UNAM.

Salinas, P. (2007). Los discursos masculinos como dispositivos de control y tensión en la configuración del liderazgo y empoderamiento femenino. Revista Estudios Feministas, 15(3), 541-562. DOI: http://dx.doi.org/10.1590/S0104026X2007000300003

Salinas, P., Reyes, C., Romaní, G. y Ziede, M. (2010). Mercado laboral femenino. Un estudio empírico, desde la perspectiva de la demanda, en la región minera de Antofagasta, Chile. Revista Innovar, 20(38), 125-139. Recuperado el 15 de noviembre 2017 de http:/ / www.redalyc.org/pdf/818/81819024010.pdf

Scott, J. (2013). El género: una categoría útil para el análisis histórico. En M. Lamas (Ed.), El género. La construcción cultural de la dife- 
rencia sexual (pp. 265-302). México: Programa Universitario de Estudios de Género: Instituto de Investigaciones sobre la Universidad y la Educación / UNAM.

Staab, S. (2012). Maternalism, breadwinner bias, and market reform: historical legacies and current reforms in Chilean social policy. Social politics, 9(6), 299-332. DOI: https://doi. org $/ 10.1093 / \mathrm{sp} / \mathrm{jxs} 010$

Stefoni, M. (2005). Supervisoras en El Teniente. Mujeres en mundos masculinos. Tesis de Magíster, Facultad de Ciencias Sociales, Universidad de Chile. Recuperado el 15 de noviembre 2017 de http://www.tesis.uchile.cl/tesis/uchile/2005/ stefoni_m/sources/stefoni_m.pdf

Tobío, C. (2012). Cuidado e identidad de género. De las madres que trabajan a los hombres que cuidan. Revista Internacional de Sociología, 70(2), 399-422. DOI: http://dx.doi.org/10.3989/ ris.2010.08.26

Tomei, M. (2006). El nexo entre discriminación e igualdad de género en el trabajo. Algunas consideraciones conceptuales y de políticas. En L. Abramo (Ed.), Trabajo decente y equidad de género en América Latina, pp. 233-262). Santiago: Oficina Internacional del Trabajo (OIT). Recuperado el 15 de noviembre 2017 de http://www.institutouejn.nqnwebs.com/documentos/genero/Trabajo $\% 20$ decente $\% 20 \mathrm{y} \% 20$ equidad $\% 20$ de $\% 20 \mathrm{~g} \% \mathrm{C} 3 \%$ A9nero\%20en\%20America\%20Latina.pdf

Troncoso, R. (2014). Proceso de subjetivación de género en las trabajadoras del cobre en Chile. El caso de la división El Teniente. Revista Temas Sociológicos, 18, 63-98. Recuperado el 15 de noviembre 2017 de http://ediciones.ucsh.cl/ojs/index. php?journal $=$ TSUCSH\&page $=$ article\&op $=$ view $\&$ path $\%$ $5 \mathrm{~B} \% 5 \mathrm{D}=717 \&$ path $\% 5 \mathrm{~B} \% 5 \mathrm{D}=586$

Wajcman, J. (1991). Feminism Confronts Technology. University Park: The Pennsylvania State University Press.

Yáñez, S. (2004). La flexibilidad laboral como nuevo eje de la producción y la reproducción. En R. Todaro y S. Yáñez (Eds.) El trabajo se transforma: Relaciones de producción y relaciones de género, (pp. 35-73). Santiago, Chile: Centro de Estudios de la Mujer.

Yopo, M. (2016). El tiempo de las mujeres en Chile: repensar la agencia. Revista de Estudios Sociales, 57, 100-109. DOI: https://doi. org $/ 10.7440 /$ res 57.2016 .08 